\title{
SPubs Súnde
}

\section{Tratamento de câncer em gestantes: Estudo de Revisão de Relatos de Casos}

\author{
Treatment of cancer in pregnant women: a case report review study
}

\author{
Cleide Silva Menezes $^{1^{\bullet}}{ }^{\ominus}$, Aline Amaral do Nascimento ${ }^{1}{ }^{\ominus}$, Lívia Cristina Alves ${ }^{1}{ }^{\ominus}$, Marta Reis \\ Correia $^{1} \bullet$, Claudinei Alves Santana ${ }^{2} \bullet$
}

${ }^{1}$ Centro Universitário SENAC, São Paulo, São Paulo, Brasil. ${ }^{2}$ Docente do Centro Universitário SENAC, São Paulo, São Paulo, Brasil. *Autor para correspondência: E-mail: cleidemenezesantos@gmail.com

\begin{abstract}
Resumo: Introdução: Câncer é um conjunto com mais de 100 patologias, que possuem crescimento desordenado, invadindo tecidos e órgãos, e são denominados de acordo com o tipo de célula e tecido em que se iniciam. O diagnóstico de câncer durante a gravidez, é considerado uma situação delicada, por gerar sofrimento a paciente e aos familiares e desafiadora aos profissionais de saúde por ser evento raro. $\mathrm{O}$ trabalho tem como objetivo, apresentar os tratamentos farmacológicos e condutas adotadas no câncer em gestantes, através de artigos de relatos de caso. Revisão: A pesquisa foi realizada a partir de buscas nas bases de dados Pubmed e Scielo, com as palavras-chave em inglês, pregnancy, cervical cancer, vulvar cancer, ovarian cancer e endometrial cancer, e seus respectivos termos em português e espanhol. Sendo o período de busca de 1 de maio de 2019 a 28 de fevereiro de 2020, sendo considerados válidos artigos publicados entre 2005 a 2019, usando como critério de inclusão e exclusão os resumos, os resultados das pesquisas de 8 relatos de casos. Discussão e considerações finais: Sugere que o tratamento adotado, siga os mesmos critérios das não gestantes, porém a pouca literatura dificulta a escolha da melhor terapêutica e demonstra a necessidade de uma equipe multiprofissional, como também a importância do farmacêutico na escolha da terapêutica mais adequada, de acordo com o estadiamento da gestante.
\end{abstract}

Palavras-chave: gestante, câncer, quimioterapia, relatos de casos.

\begin{abstract}
Introduction: Cancer is a group with more than 100 pathologies, which have disordered growth, invading tissues and organs, and are named according to the type of cell and tissue in which they start. The diagnosis of cancer during pregnancy is considered a delicate situation, because it generates suffering for the patient and family and challenging health professionals because it is a rare event. The work aims to present the pharmacological treatments and conducts adopted in cancer in pregnant women, through articles of case reports. Review: The research was conducted based on searches in the Pubmed and Scielo databases, with the keywords in English, pregnancy, cervical cancer, vulvar cancer, ovarian cancer and endometrial cancer, and their respective terms in Portuguese and Spanish. Being the search period from May 1, 2019 to February 28, 2020, articles published between 2005 to 2019 are considered valid, using abstracts and research results from 8 case reports as inclusion and exclusion criteria. Discussion and final considerations: It suggests that the treatment adopted, follow the same criteria as non-pregnant women, however the little literature makes it difficult to choose the best therapy and demonstrates the need for a multidisciplinary team, as well as the importance of the pharmacist in choosing the most appropriate therapy, according to the pregnant woman's staging.
\end{abstract}

Keywords: pregnant woman, cancer, chemotherapy, case reports.

\section{Introdução}

Câncer é um conjunto com mais de 100 patologias, que possuem crescimento desordenado, invadindo tecidos e órgãos, e são denominados de acordo com o tipo de célula e tecido em que se iniciam, não havendo uma causa definida, podendo ser de origem externa, como causas relacionadas a hábitos de vida e meio ambiente ou internas, como hormônios e condições imunológicas com a capacidade de invadir tecidos e órgãos vizinhos ou distantes, o que é chamado de metástase (https://www.inca.gov.br/causas-e-prevencao/oque-causa-cancer).

O diagnóstico de câncer durante a gravidez, é considerado uma situação delicada, por gerar sofrimento a paciente e aos familiares e desafiadora aos profissionais de saúde, devido à pouca pesquisa e literatura abordando o tema considerado um evento raro, com ocorrência de 1 caso para 1000 gestantes (Loibl et al., 2006). 
Dentre os tipos mais incidentes estão câncer de mama, cervical, ovário, linfoma e melanoma, sendo a abordagem terapêutica realizada de acordo com a extensão da doença, período gestacional, como também uma abordagem multidisciplinar, incluindo obstetras, ginecologistas, especialistas em medicina materno fetal, oncologistas, neonatologistas, farmacêuticos, assistentes sociais e psicólogos (Salani et al., 2014)

Parte da terapêutica para esta condição de saúde também é a utilização de fármacos antineoplásicos, porém este conhecimento está limitado, tanto para o início da terapia como a escolha do medicamento e dose apropriada, sabe se que alterações fisiológicas na gestação podem afetar de modo expressivo os medicamentos, especialmente no segundo e terceiro trimestre da gestação (Esposito et al., 2016).

Sabe-se que os fármacos, com alta solubilidade lipídica, baixo peso molecular e baixa taxa de ligação às proteínas plasmáticas têm maior transferência placentária, e as monografias de vários medicamentos anticâncer afirmam que seu uso é contraindicado, como regra geral, durante a gravidez, segundo o FDA, praticamente todos estão na categoria D e X de risco (Miyamoto et al., 2016).

Um estudo de caso de câncer de mama (local e ano) na gestação, sugere, que o tratamento deva seguir as mesmas diretrizes para não grávidas, sendo a quimioterapia indicada após a organogênese e interrompida por volta da $35^{\text {a }}$ semana, até o parto, evitando a mielossupressão, enquanto que a terapia hormonal, a radioterapia e a terapia com anticorpos monoclonais anti HER2, não estão indicadas em nenhuma fase da gestação (Alfasi \& Ben-Aharon, 2019)

Os tratamentos sistêmicos de câncer hematológico e de mama em gestantes e não gestantes, incluem também as antraciclinas, e estudos mostram que esses fármacos possuem níveis de segurança aceitáveis, porém, alerta sobre os riscos relacionados a dose e à toxicidade cardíaca fetal, destacando a teratogenicidade da Idarubicina, e seu uso apenas quando não houver outra opção (Framarino-Dei-Malatesta et al., 2017).

Em uma revisão sistemática e meta-análise, realizada sobre o câncer cervical, na gestação, a proposta é a utilização de cisplatina, em monoterapia, a fim de diminuir os efeitos adversos da quimioterapia (Song et al., 2018).

Dentro da escolha dos tratamentos a serem realizados, a figura do Farmacêutico Clínico é de extrema importância na equipe multiprofissional, atribuindo a este profissional a seleção, padronização de medicamentos e materiais e manipulação dos agentes antineoplásicos proporcionando qualidade a farmacoterapia empregada através da análise da prescrição médica em conjunto da avaliação dos agentes antineoplásicos por possuírem estreita janela terapêutica que expõe os pacientes a riscos (http://www.cff.org.br/sistemas/geral/revista/pdf/70/encarte pb70.pdf). O trabalho tem como proposta apresentar os tratamentos farmacológicos e condutas médicas adotadas no câncer em gestantes e desfechos clínicos nas crianças através de artigos de relatos de caso.

\section{Revisão}

Trata-se de um artigo de revisão da literatura através da busca nas bases de dados Pubmed e Scielo com as palavras-chave em inglês Pregnancy, Cervical cancer, Vulvar cancer, Ovarian cancer e Endometrial câncer e respectivos termos em português e espanhol. O período da busca foi de 1 maio de 2019 a 28 de fevereiro 2020 sendo considerados válidos artigos publicados entre 2005 a 2019.

A partir da metodologia adotada foram encontrados 35 artigos. Destes foram excluídos 17 artigos que abordavam o tema câncer em pacientes não grávidas. Foram incluídos desta forma 19 artigos com resumos que abordavam o tema câncer durante a gestação.

Tabela 1. Estudos de relato de caso de câncer em gestantes segundo nacionalidade e ano de publicação.

\begin{tabular}{ccc}
\hline Artigo & Nacionalidade & Ano \\
\hline Câncer de mama gestacional: relato de caso & Brasil & 2017 \\
\hline $\begin{array}{c}\text { Tratamento quimioterapêutico do câncer colorretal na gravidez: relato } \\
\text { de caso }\end{array}$ & Arábia Saudita & 2015 \\
\hline Câncer de mama e gravidez: relato de casos & Brasil & 2012 \\
\hline $\begin{array}{c}\text { Quimioterapia neoadjuvante seguida de cirurgia radical em paciente } \\
\text { grávida com câncer de colo de útero: relato de caso e revisão de literatura }\end{array}$ & Brasil & 2011 \\
\hline Câncer de reto durante gestação: relato de caso e revisão de literatura & Brasil & 2011 \\
\hline Neoplasia "borderline” do ovário e gestação: relato de caso & Brasil & 2006 \\
\hline
\end{tabular}


A falta de literatura e as questões éticas, abordando o tema, dificultam a escolha do tratamento farmacológico, sendo apresentado os relatos de casos de gestantes com câncer em diversos períodos gestacionais e seus respectivos desfechos até o parto.

Caso 1: 2017. Mulher, 34 anos, $19^{\text {a }}$ semana de gestação, admitida no pronto atendimento de um hospital em Bogotá com queixas de dor lombossacra esquerda e aumento progressivo. Os exames mostraram a princípio anemia aguda com hemoglobina $6,4 \mathrm{~g} / \mathrm{dl}$ e melena, dessa forma realizou-se o exame de endoscopia que apresentou achados microscópicos compatíveis com carcinoma gástrico Borman III. O diagnóstico foi realizado a $19^{\mathrm{a}}$ semana e 3 dias de gestação. Outros estudos foram realizados a fim de estadiar a doença neoplásica. $\mathrm{O}$ exame de ressonância magnética evidenciou massa ovariana esquerda medindo $10 \times 10 \mathrm{~cm} \mathrm{e}$ metástases ósseas no fêmur proximal e sacro contraindicando o parto vaginal. Diante do desejo da paciente de continuar a gestação, e estágio avançado do câncer, a equipe médica decidiu por um manejo paliativo com esquema quimioterápico FOL-FIRE, 5 ciclos por semana inicialmente às $22^{\mathrm{a}}$ semanas e 6 dias de gestação. Em razão de o feto apresentar peso de $1492 \mathrm{~g}$ às $33^{\mathrm{a}}$ semanas e 5 dias foi indicada a cesariana e a paciente deu à luz a um recém-nascido do sexo masculino. Após um mês em acompanhamento neonatal básico no hospital e progressão da doença da paciente, mãe e filho foram liberados para acomodação em casa, porém passados dois meses a paciente veio a óbito devido a doença (Fory et al., 2017).

Caso 2: 2015. Saudita 33 anos, diagnosticada com câncer colo retal com $11^{\text {a }}$ semana de gestação, concordou com término da gravidez, sendo realizada intervenção abortiva com metotrexato intravaginal que não foi realizada com sucesso. A paciente então decidiu prosseguir com a gestação iniciando quimioterapia adjuvante bimestralmente com folfox 6 (oxaliplatina, leucovorina, fluorouracil) na $22^{\text {a }}$ semana e após seis ciclos houve o adiamento do tratamento para depois do parto. Na $38^{\mathrm{a}}$ semana de gestação houve a indução do parto. Tanto a RN (recém-nascido) quanto a mãe não tiveram complicações imediatas, e a criança foi acompanhada por 2 anos, e seu desenvolvimento seguiu sem complicações reconhecíveis e a mãe seguiu o tratamento pós-parto (Makoshi et al., 2015).

Caso 3: 2011. Brasileira, 43 anos, diagnosticada com câncer de mama com $32^{\text {a }}$ semanas de gestação, realizou 2 ciclos de quimioterapia de 21 dias com doxorrubicina e ciclofosfamida, retendo a continuidade do tratamento para depois do parto. $\mathrm{Na} 35^{\mathrm{a}}$ semana a paciente recebeu corticoterapia para indução da maturidade pulmonar fetal, realizada a cesariana o RN apresentou alterações anatômicas, sugestivas de Síndrome de Down, associados a idade da mãe, ambos seguiram sendo acompanhados por serviços especializados (Bastos \& Monteiro, 2012).

Caso 4: 2011. Brasileira, 35 anos, na $10^{a}$ semana de gestação recebeu o diagnóstico de carcinoma ductal invasor na mama direita. Foi realizada mastectomia radical modificada, e logo em seguida a paciente recebeu quimioterapia com 6 ciclos de doxorrubicina $60 \mathrm{mg} / \mathrm{m} 2$ e ciclofosfamida $600 \mathrm{mg} / \mathrm{m} 2$. Sendo realizada cesariana. A paciente não amamentou e foi prescrito tamoxifeno $20 \mathrm{mg} /$ dia por cinco anos, e 4 anos após a cirurgia a criança encontrava saudável (Manoel et al., 2011).

Caso 5: 2011. Brasileira, 31 anos, na $24^{\text {a }}$ semana de gestação, procurou atendimento médico na Unidade de Emergência se queixando que há 30 dias começou a apresentar afilamento do calibre das fezes e sangramento retal. Foi realizado exame proctológico, detectando lesão tumoral na parede anterior do reto com abscesso drenando secreção purulenta. Realizada biópsia que indicou adenocarcinoma moderadamente diferenciado invasivo estando presente também na parede da vagina. A equipe de obstetrícia avaliou o feto e estava em condições normais. Nos exames laboratoriais indicou anemia e na ressonância magnética mostrou um grande volume de massa anorretal concluindo grande comprometimento do reto, sendo útero e bexiga não apresentando indício de invasão do tumor. A gestação foi mantida até atingir a maturidade pulmonar do feto e quando completou $27^{\text {a }}$ semanas foi realizada o parto e o recém-nascido foi encaminhado ao berçário de alto risco. Uma semana depois do parto a paciente realizou a laparotomia, histerectomia e ressecção das áreas acometidas. A criança após os cuidados do berçário foi para o quarto e logo recebeu alta, e a mãe recebeu alta 9 dias após a cirurgia e foram realizadas radioterapia e quimioterapia adjuvantes. A paciente faleceu 26 meses depois devida metástase no fígado e pulmões (Freitas et al., 2011)

Caso 6: 2011. Brasileira, 30 anos, na $24^{\text {a }}$ semana de gestação procurou atendimento médico devido a sangramento vaginal. No exame especular foi detectado lesão tumoral sangrante no colo de útero. Foi realizada a biópsia cervical indicando carcinoma de células escamosas moderadamente diferenciado, e na ressonância magnética do abdômen indicou lesão cervical de 3 a $4 \mathrm{~cm}$, sem indícios de dilatação das vias urinárias. A paciente não tinha filho e recusou a interromper a gestação. Depois de uma minuciosa explicação sobre os riscos envolvendo a paciente e o feto, a equipe médica juntamente com a gestante optaram em iniciar a quimioterapia neoadjuvante a cada 21 dias com vincristina $1 \mathrm{mg} / \mathrm{m} 2$ e cisplatina $75 \mathrm{mg} / \mathrm{m} 2$ e a cesárea ficou planejada em conjunto à cirurgia radical. Foram ministrados 4 ciclos de quimioterapia bem tolerados e com 
baixa toxicidade, e logo depois do primeiro ciclo, o sangramento vaginal cessou. Foi realizada a ultrassonografia na $28^{\mathrm{a}}$ semana de gestação para verificar o crescimento do feto e o resultado foi adequado para o tempo gestacional. Após o terceiro ciclo da quimioterapia obteve a resposta clínica completa e ficou programada para a $38^{\mathrm{a}}$ semana de gestação a cesárea. Na $37^{\mathrm{a}}$ semana a paciente entrou em trabalho de parto nascendo uma criança sem irregularidade pesando 2.450 gramas (peso abaixo do estimado para tempo de gestação). Três dias após o parto foi realizada a cirurgia para histerectomia radical com linfadenectomia pélvica e paraaórtica sem anormalidade e ambos tiveram alta 5 dias após a cirurgia em bom estado geral. Não foi necessária a prescrição de tratamento adjuvante e a paciente foi orientada a realizar consultas a cada três meses com a equipo de oncologia para a realização de exames para acompanhamento da evolução do quadro e após 12 meses de acompanhamento não houve indícios da doença e a criança estava se desenvolvendo normalmente (Fonseca et al., 2011).

Caso 7: 2006. Brasileira, 35 anos, na $19^{\mathrm{a}}$ semana de gestação foi diagnosticada com cistadenocarcinoma seroso "borderline" ou de baixo potencial de malignidade. Na $31^{\text {a }}$ semana de gestação foi realizada a cirurgia via laparotomia para retirada da formação cística. No pós-operatório não houve ocorrência e a gestação seguiu até a $41^{\text {a }}$ semana, onde houve um quadro hipertensivo e foi preciso induzir o trabalho de parto. Realizado parto normal, sem irregularidade, e o bebê nascendo saudável. Posteriormente foi realizado histerectomia total do abdômen, biópsia de omento maior e todos os resultados foram negativos (Souza et al., 2006).

Caso 8: 2005. Brasileira, 26 anos, na $17^{\mathrm{a}}$ semana de gestação foi diagnosticada com tumor desmóide na face anterior do abdômen, com crescimento do tumor controlado no decorrer da gestação, no entanto, houve crescimento significativo no último trimestre da gestação. A criança nasceu por meio de cesariana e sem anormalidade. Devido ao diagnóstico de neoplasia maligna e pelo risco de disseminação, não foi feito biópsia, e os médicos optaram por ressecção cirúrgica em monoblocos do tumor. A paciente teve alta no $4^{\text {a }}$ dia após a última cirurgia apresentando uma boa evolução (Priolli et al., 2005).

Segundo o FDA os antineoplásicos pertencem por via de regra à categoria $\mathrm{D}$ e X, sendo D- risco para o feto, entretanto para a gestante os benefícios podem ser maiores, categoria $\mathrm{X}$ - riscos para o feto que ultrapassam os benefícios. Os esquemas mostraram-se seguros quando a utilização foi realizada após o primeiro trimestre da gestação e em sua maioria não houve intercorrências com o feto. (Monteiro et al., 2013).

Tabela 2. Caracterização das pacientes que desenvolveram câncer na gestação em relação a idade, fármacos utilizados, classificação de risco e principais reações adversas possíveis.

\begin{tabular}{|c|c|c|c|}
\hline Paciente & Fármacos Utilizados & $\begin{array}{l}\text { Class } \\
\text { Risco }\end{array}$ & Reações adversas \\
\hline \multirow{4}{*}{$\begin{array}{l}\text { Saudita, } 33 \\
\text { anos }\end{array}$} & Oxaliplatina, & $\mathrm{D}$ & taquicardia, edema, pancreatite aguda, \\
\hline & $\begin{array}{l}\text { Leucovorina (ácido } \\
\text { folínico) }\end{array}$ & $\mathrm{C}$ & diarreia, estomatite, mielossupressão. \\
\hline & Metotrexato & $\mathrm{X}$ & hemorragia, cirrose, disfunção renal, leucopenia, etc. \\
\hline & Fluorouracil & $\mathrm{D}$ & $\begin{array}{l}\text { leucopenia, neutropenia, anemia, trombocitopenia. anorexia, náuseas e vômitos, } \\
\text { estomatite. }\end{array}$ \\
\hline \multirow{2}{*}{$\begin{array}{l}\text { Brasileira, } 43 \\
\text { anos }\end{array}$} & Doxorrubicina & $\mathrm{D}$ & estomatite, diarreia, vômito, náusea, infecção, sepse \\
\hline & Ciclofosfamida & B & náusea, vômito, constipação, dor abdominal, leucopenia, trombocitopenia. \\
\hline \multirow{2}{*}{$\begin{array}{l}\text { Brasileira, } 34 \\
\quad \text { anos }\end{array}$} & Vincristina & $\mathrm{D}$ & $\begin{array}{l}\text { alopecia, distúrbios neuromusculares, neurotoxicidade, anafilaxia, edema, náusea, } \\
\text { vômito, cólica abdominal. }\end{array}$ \\
\hline & Cisplatina & $\mathrm{D}$ & $\begin{array}{c}\text { nefrotoxicidade, ototoxicidade (zumbido, perda auditiva), neurotoxicidade, } \\
\text { toxicidade ocular, miolesupressão, náusea, vômito, anorexia, diarréia. }\end{array}$ \\
\hline $\begin{array}{l}\text { Brasileira, } 35 \\
\quad \text { anos }\end{array}$ & Tamoxifeno & $\mathrm{D}$ & $\begin{array}{l}\text { náusea, retenção de líquidos, sangramento vaginal, erupção cutânea, fogacho, } \\
\text { fadiga, anemia, catarata, alopecia, cefaleia. }\end{array}$ \\
\hline
\end{tabular}

\section{Considerações finais}

O tratamento oncológico em pacientes gestantes continua sendo um grande desafio para a equipe de saúde.

Na comparação das bulas dos medicamentos com os relatos de casos, verifica se que as bulas deixam claro que o risco de uso em gestantes está entre D e X, ou seja, alto risco por falta de estudos, gerando um dilema para os profissionais envolvidos, de qual conduta tomar. 
O câncer durante a gestação é um evento raro e grave, porem tratável, e o uso dos quimioterápicos a partir do segundo trimestre de gestação, tem se mostrado seguros, para mãe e não tem apresentado danos aos RN, porém a pouco literatura, dificulta a escolha de qual conduta a ser tomada, tornando evidente a necessidade do profissional Farmacêutico, compondo a equipe multiprofissional, auxiliando nas tomadas de decisões em relação à melhor terapêutica.

\section{Referências}

Alfasi, A., \& Ben-Aharon, I. 2019. Breast Cancer during Pregnancy-Current Paradigms, Paths to Explore. Cancers, 11(11), 1669.

Bastos, F. A., \& Monteiro, I. M. 2012. Câncer de mama e gestação: relato de caso. Revista Paraense de Medicina.

Esposito, S., Tenconi, R., Preti, V., Groppali, E., \& Principi, N. 2016. Chemotherapy against cancer during pregnancy: A systematic review on neonatal outcomes. Medicine, 95(38).

Fonseca, A. J. D., Dalla-Benetta, A. C., Ferreira, L. P., Martins, C. R. N., \& Lins, C. D. M. 2011. Quimioterapia neoadjuvante seguida de cirurgia radical em paciente grávida com câncer de colo de útero: relato de caso e revisão de literatura. Revista Brasileira de Ginecologia e Obstetrícia, 33(1), 43-48.

Fory, J. A., Arenas, V., Carmona, V., \& Llanos, J. 2017. Endocarcinoma gástrico y embarazo reporte de dos casos y revisión de la literatura. Revista Med, 25(2), 63-73.

Framarino-dei-Malatesta, M., Sammartino, P., \& Napoli, A. 2017. Does anthracycline-based chemotherapy in pregnant women with cancer offer safe cardiac and neurodevelopmental outcomes for the developing fetus?. BMC Cancer, 17(1), 777.

Freitas, A. H. A., Madeira, H., Fonseca, L. M. D., \& Castro, E. V. D. 2011. Câncer de reto durante gestação: relato de caso e revisão da literatura. Revista Brasileira de Coloproctologia, 31(1), 81-84.

Loibl, S., Von Minckwitz, G., Gwyn, K., Ellis, P., Blohmer, J. U., Schlegelberger, B., ... \& Klingebiel, T. 2006. Breast carcinoma during pregnancy: international recommendations from an expert meeting. Cancer: Interdisciplinary International Journal of the American Cancer Society, 106(2), 237-246.

Makoshi, Z., Perrott, C., Al-Khatani, K., \& Al-Mohaisen, F. 2015. Chemotherapeutic treatment of colorectal cancer in pregnancy: case report. Journal of medical case reports, 9(1), 140.

Manoel, W. J., Mühlbeier, D. F. M., Valadares, F. D., Alves, L. G., Abreu, D. C. B., Paula, É. C. D., ... \& Saddi, V. A. 2011. Câncer de mama e gravidez: relato de caso.

Miyamoto, S., Yamada, M., Kasai, Y., Miyauchi, A., \& Andoh, K. 2016. Anticancer drugs during pregnancy. Japanese journal of clinical oncology, 46(9), 795-804.

Monteiro, D. L. M., Trajano, A. J. B., Menezes, D. C. S., Silveira, N. L. M., Magalhães, A. C., de Miranda, F. R. D., \& Caldas, B. (2013). Câncer de mama na gravidez e quimioterapia: revisão sistemática. Revista da Associação Médica Brasileira, 59(2), 174-180.

Priolli, D. G., Martinez, C. A. R., Mazzini, D. L. S., Souza, C. A. F. D., Piovesan, H., \& Nonose, R. 2005. Tumor desmóide da parede abdominal durante a gravidez: relato de caso. Revista Brasileira de Ginecologia e Obstetrícia, 27(5), 283-288.

Salani, R., Billingsley, C. C., \& Crafton, S. M. 2014. Cancer and pregnancy: an overview for obstetricians and gynecologists. American journal of obstetrics and gynecology, 211(1), 7-14.

Song, Y., Liu, Y., Lin, M., Sheng, B., \& Zhu, X. 2019. Efficacy of neoadjuvant platinum-based chemotherapy during the second and third trimester of pregnancy in women with cervical cancer: an updated systematic review and meta-analysis. Drug Design, Development and Therapy, 13, 79.

Souza, L. R. M. F., Marques, R. M., Nicolau, S. M., Gonçalves, W. J., Neto, C. S., Goldman, S. M., \& Szejnfeld, J. 2006. Neoplasia “borderline” do ovário e gestação: relato de caso.

\section{Minicurrículo}

Cleide Silva Menezes. Possui especialização em Farmácia Clinica e Hospitalar pelo Centro Universitário SENAC/SP (2020) e graduação em Farmácia pela Universidade Brasil (2016). Atualmente é farmacêutico SPDM - associação paulista para o desenvolvimento. Tem experiencia na área de farmácia clinica com ênfase em saúde pública e coletiva. 
Aline Amaral do Nascimento. Possui graduação em Farmácia e Bioquímica pela universidade nove de julho (2016) e especialização em Farmácia Clínica e Hospitalar pelo Serviço Nacional de Aprendizagem Comercial SP (2020). Atualmente Farmacêutica responsável técnica da Raia Drogasil.

Lívia Cristina Alves. Possui graduação em Farmácia pela Universidade Anhanguera de São Paulo (2012). Atualmente é farmacêutica responsável na rede de farmácia Drogaria Campeã; concluiu o curso de Farmácia Clínica e Hospitalar pelo Senac (SP).

Marta Reis Correia. Possui graduação em Farmácia pela Universidade Paulista (2015). Atualmente é Farmacêutica Responsável da Raia Drogasil. Tem experiência na área de Farmácia.

Claudinei Alves Santana. Possui graduação em Farmácia Bioquímica e Homeopatia pela Universidade São Judas Tadeu (2004). Especialização em Farmácia Hospitalar pela Faculdade Oswaldo Cruz (2005) e Planejamento Educacional e Docência do Ensino Superior pela ESAB (2010). Especialização Multiprofissional em Oncologia pelo Hospital Sírio Libanês (2019). Mestre em Ciências Médicas pela Faculdade de Medicina da USP (2014). Farmacêutico responsável pelo Serviço de Extensão ao Atendimento de Pacientes HIV/AIDS do HC-FMUSP (2005 -2013). Docente da Pós-Graduação SENAC/SP. Docente da Pós Graduação ICTQ. Experiência em Manipulação, Acompanhamento clínico e Dispensação de Quimioterápicos. Experiência em Farmácia Clínica, Ensino, Assistência a Pacientes HIV/AIDS.

Como citar: Menezes, C.S., Nascimento, A.A., Alves, L.C., Correia, M.R., \& Santana, C.A. 2020. Tratamento de câncer em gestantes: Estudo de Revisão de Relatos de Casos. Pubsaúde, 4, a075. DOI: https://dx.doi.org/10.31533/pubsaude4.a075

Recebido: 15 set. 2020

Revisado e aceito: 22 set. 2020.

Conflito de interesse: os autores declaram, em relação aos produtos e companhias descritos nesse artigo, não ter interesses associativos, comerciais, de propriedade ou financeiros que representem conflito de interesse.

Licenciamento: Este artigo é publicado na modalidade Acesso Aberto sob a licença Creative Commons Atribuição 4.0 (CC-BY 4.0). 